- 研究报告・

\title{
中国瓢虫体型的地理分异及其与环境因子的关系
}

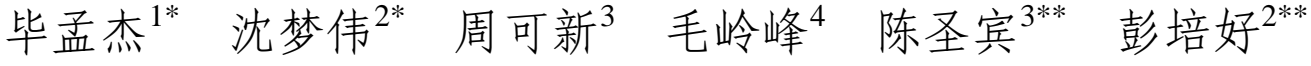 \\ 1 (成都理工大学地球科学学院, 成都 610059) \\ 2 (成都理工大学旅游与城乡规划学院, 成都 610059) \\ 3 (环境保护部南京环境科学研究所, 南京 210042) \\ 4 (Department of Renewable Resources, University of Alberta, Edmonton, Alberta, Canada T6G 2H1)
}

\begin{abstract}
摘要: 生物体的外在形态(个体大小及异速生长)不仅是分类学的重要依据, 也是影响生物与环境相互作用的重要 功能性状。昆虫体型的地理分异及其机制是昆虫生物地理学的重要基础。本文基于中国省级行政区的㼼虫体型、 分布和环境因子数据, 运用相关和回归分析, 探索了中国慓虫体型(体长、体宽与长宽比)的纬度地带性及其与环境 因子的关系。为了确定营养级对漂虫体型特征的影响, 我们对全部漂虫、植食性漂虫和捕食性瓢虫分别进行分析。 研究结果表明: (1)㼼虫的体型大小具有显著的纬度地带性, 即纬度越高, 慓虫的体长或体宽越大, 符合贝格曼法 则。与温度相关的环境因子是导致漂虫体型大小地理分异的主要环境因素, 因为瓢虫以成虫越冬, 体型越大则能 量储存越多, 冬眠期间的耐饥力越强; (2)由于食性和食物空间分布的差异, 在各个纬度上, 植食性漂虫体型总是 大于捕食性慓虫; 捕食性漂虫的长宽比与纬度呈显著正相关, 即纬度越高, 慓虫体型越狭长; 而植食性漂虫的长 宽比与纬度没有显著的相关性; 捕食性㼼虫的长宽比与年降水量呈显著负相关, 因为年降水量降低导致植被的斑 块化增加, 引起捕食对象分布更为分散, 从而要求漂虫具有更强的飞行能力, 即更大的长宽比。综上所述, 在省级 尺度上, 温度和降水分别主要影响瓢虫的大小和长宽比, 其效应随营养级(食性)的变化而变化。
\end{abstract}

关键词：慓虫，体型，环境因子，体长，体宽，长宽比

\section{Geographical variance of ladybird morphology and environmental cor- relates in China}

\author{
Mengjie $\mathrm{Bi}^{1^{*}}$, Mengwei Shen ${ }^{2 *}$, Kexin Zhou ${ }^{3}$, Lingfeng $\mathrm{Mao}^{4}$, Shengbin Chen ${ }^{3 * *}$, Peihao Peng ${ }^{2 * *}$ \\ 1 College of Earth Sciences, Chengdu University of Technology, Chengdu 610059 \\ 2 College of Tourism and Urban-Rural Planning, Chengdu University of Technology, Chengdu 610059 \\ 3 Nanjing Institute of Environment Sciences, Ministry of Environmental Protection, Nanjing 210042 \\ 4 Department of Renewable Resources, University of Alberta, Edmonton, Alberta, Canada T6G $2 \mathrm{H} 1$
}

\begin{abstract}
The extent shape of organisms (body size and the allometry of specific parts) is not only the base for taxonomy, but also the key functional traits for understanding the interaction between organisms and environmental conditions. The geographical variance of body size, allometry, and the underlying mechanisms are fundamental for insect biogeography, but have not been fully documented. In this paper, we compiled data on body size (body length and body width), aspect ratio (body length/body width), and distribution of ladybirds in 28 provinces in China. Meanwhile, we obtained environmental variables representing temperature and precipitation from freely available data. To explore the spatial variance and environmental correlates of body size and aspect ratio in different species groups with various trophic positions, correlation and regression analyses were performed separately on herbivorous and predaceous ladybirds. Our results show that there is evident latitudinal gradient for body size of ladybirds, i.e., body length or width increases with increasing latitude. This is consistent with Bergmann's rule. Temperature-related variables are the main drivers of geographic variance of body size, because ladybirds usually overwinter as adults, and those with larger
\end{abstract}

收稿日期: 2015-08-04; 接受日期: 2015-09-15

基金项目：国家科技支撑计划(2012BAC01B08)和国家环境保护公益性行业科研专项(201409055)

*共同第一作者 Equally contributed to this paper

** 共同通讯作者 Co-authors for correspondence. E-mail: chainpin@126.com, peihaop@163.com 
body size have more fat and therefore are more resistant to starvation in winter. Herbivorous ladybirds are always larger than predaceous ladybirds in terms of body length and width along the latitudinal gradients. This is due to discrepancies in food and nitrogen concentrations, with predaceous ladybirds having higher nitrogen concentrations than herbivorous ladybirds. Aspect ratio increases significantly with latitude for predaceous ladybirds, but not for herbivorous ladybirds. This may be explained by the high spatial dispersion of prey for predaceous ladybirds, which are further influenced by their own host plants. The most important environmental variables controlling geographic variance in aspect ratio of predaceous ladybirds are precipitation-related, but not temperature-related variables. This is because decreasing precipitation will lead to increasing patchiness of vegetation and subsequently prey for predaceous ladybirds. Increasing aspect ratio and accordingly flying ability is an adaptive response under these conditions. We concluded that, at the provincial scale in China, temperature-related and precipitation-related variables are the main determinant factors for body size and aspect ratio of herbivorous and predaceous ladybirds, respectively; and their magnitude of effects on body shape depends on the trophic positions.

Key words: ladybirds, body size, environmental factor, body length, body width, aspect ratio

生物体的外在形态不仅是分类学的重要依据, 也是影响生物与环境相互作用的重要功能性状 (Chown \& Gaston, 2010)。形态功能性状不仅包括体 重、体长、体积等表示个体大小的指标, 也包括生 物体各个组织或器官之间的异速生长关系(Barbault, 1988; Azevedo et al., 1998)。深入理解种内或 种间形态功能性状在地理尺度上的分异特征及其 与生物和非生物因子的关系, 是理解物种地理分布 和预测气候变化对生物多样性影响的重要基础 (Chown \& Gaston, 2010; Gardner et al., 2011; Comont et al., 2012; 匡先矩等, 2015)。

体型大小是生物体最重要的特征之一，几乎影 响生物体的各个方面, 如繁殖力、代谢速率、巢穴 大小和地理分布范围等(Peters, 1983; Blanckenhorn \& Demont, 2004; Brown et al., 2004; Chown \& Gaston, 2010)。目前最为广泛接受的关于动物个体大小 的空间格局的理论是贝格曼法则(Bergmann's rule), 它认为动物的体型常随纬度 (或海拔)升高而变大 (Bergmann, 1847; Shelomi, 2012; 匡先矩等, 2015)。 但是这一法则最初是针对鸟类和哺乳类等温血动 物提出的, 并未对冷血动物体型大小随纬度的变化 趋势作出预测(Blackburn et al., 1999)。对哺乳动物 而言, 其机理解释是个体越大则表面积与体积的比 例越小, 有助于在寒冷气候下保持体温而得以存 活。已有研究表明, 有些昆虫类群个体大小随纬度 升高而增加, 符合贝格曼法则; 但同时也有很多相 反的案例，符合反贝格曼法则(Park，1949; Blanckenhorn \& Demont, 2004; Shelomi, 2012)。反贝格曼
法则认为随着纬度的升高, 生长季缩短, 动物的发 育时间相应减少，因而生物体型有变短变小的趋势 (Park, 1949; Masakis, 1967; Nylin \& Svärd, 1991; Mousseau, 1997)。贝格曼法则的主要解释机理集中 在温度, 特别是冬季低温对生物体形态的影响; 对 昆虫而言, 高纬度地区动物个体增大主要是受低温 的制约; 而反贝格曼法则主要从生长季长短的角度 解释, 认为个体变小是一种适应性变化; 对昆虫而 言，一般个体较大、发育时间较长的物种, 更可能 符合反贝格曼法则，而个体较小、发育时间较短的 物种，更可能符合贝格曼法则 (Blanckenhorn \& Demont, 2004)。也就是说，世代时间与生长季的相 对长短可能共同决定了其体型大小随纬度的变化 是符合贝格曼法则还是反贝格曼法则。

生物体的形状，或各器官的相对生长关系也与 随纬度或海拔变化的气候因子具有一定的关系。如 艾伦法则(Allen's rule)认为，在恒温动物中，同种的 个体或近缘的异种之间, 生活在寒冷地区的, 其 耳、吻、首、肢、翼和尾等突出的部分具有缩短的 适应性倾向(Allen，1877)。对于可飞行的昆虫而言， 与飞行能力(如持续时间、飞行速度和能量效率)相 关的功能性状可能也存在一定的地理分异, 但却很 少受到关注(Vandewoestijne \& Van Dyck, 2011)。昆 虫一般体型较小, 无法产生足够的代谢热量调节体 温, 因此翅膀振动频率随外界温度线性增加 (Blanckenhorn \& Demont, 2004)。由于升力与翅膀振 动频率的平方呈线性正相关, 温度降低, 则升力随 之降低(Azevedo et al., 1998)。昆虫的飞行也受自身 
体型特征, 如体重、翅膀面积、翅膀载荷(体重与翅 膀面积的比例)和展弦比(翅膀长度与宽度的比例) 的影响(Hoffmann \& Shirriffs, 2002)。因此, 昆虫可 能需要通过提高展弦比和降低翅膀载荷弥补低温 下翅膀振动频率降低对飞行的不利影响(Azevedo et al., 1998; Blanckenhorn \& Demont, 2004; Vandewoestijne \& Van Dyck, 2011)。

慓虫是隶属于鞘翅目慓虫科昆虫的通称, 多数 为漂形, 虫体周缘近于卵圆形, 鞘翅肩角部分呈半 球形拱起。但在慓虫科内体型还有各种变异, 主要 有3个类型: 漂型、突肩型、长足型。漂虫的大小有 明显的差异, 在小艳漂虫亚科和小毛慓虫亚科中, 大部分种类体长为 $1.5-4.0 \mathrm{~mm}$, 但也有体长为 $0.8-$ $1.5 \mathrm{~mm}$ 的微小种类; 在慓虫亚科和食植漂虫亚科 中, 有体长超过 $10 \mathrm{~mm}$ 的大型种, 其中突肩慓虫族 中的个别种类体长甚至可达 $17 \mathrm{~mm}$ (庞虹等, 2004)。

我们采用体长和体宽作为个体大小的度量 (Rogers et al., 1976; Gruner, 2003), 以长宽比代表展 弦比, 基于中国㼼虫体型和省级行政区分布数据, 探讨漂虫种间平均体型特征的地理分异特征及其 与环境因子的关系。根据前文综述, 我们提出以下 假设: (1)漂虫个体大小随纬度增加, 符合贝格曼法 则, 且主要影响因素为温度; (2)慓虫展弦比会随纬 度增加而增加以弥补低温下翅膀振动频率的下降; (3)植食性和捕食性漂虫体型特征与纬度及环境因 子的关系可能有所不同。

\section{1 材料与方法}

\section{1 慓虫体型数据}

漂虫地理分布以及体长和体宽数据主要来自 《中国漂虫物种多样性及其利用》(庞虹等, 2004) 和《中国漂虫原色图鉴》(任顺祥等, 2009), 以其他 区域性漂虫区系和分类学文献为补充。由于直辖市 和特别行政区面积较小, 我们将其合并到临近的省 份, 即北京和天津合并到河北省, 上海合并到浙江 省, 重庆合并到四川省, 香港和澳门合并到广东 省。本文的基本分析单元为合并后的28个省份。

利用 3 个指标度量漂虫体型特征: 体长 $(L$, $\mathrm{mm}) 、$ 体宽 $(W, \mathrm{~mm})$ 和长宽比 $(L / W)$ 。我们根据各省 漂虫分布和形态数据计算上述 3 个指标的几何平均 值, 以降低极值的影响(Quinn \& Keough, 2002)。体 长和体宽不仅是个体大小的度量(Rogers et al.,
1976; Gruner, 2003), 同时也在很大程度上分别决定 了翅膀的长度和宽度, 因此可将长宽比视为展弦比 的粗略度量。

为了对比不同食性瓢虫亚科体型的地理格局 及其与环境因素相关性的差异, 我们还分别计算了 各省植食性漂虫和捕食性瓢虫的上述3个指标。其 中植食性㼼虫仅包括了植食性漂虫亚科, 而捕食性 㼼虫包括了小艳漂虫亚科、小毛㼼虫亚科、刻眼㼼 虫亚科、隐胫慓虫亚科、盔唇慓虫亚科、显盾漂虫 亚科、红㼼虫亚科和四节漂虫亚科(庞虹, 2001)。㼼 虫亚科的食菌漂虫族以真菌为食, 但种类较少, 未 进行单独研究。全部漂虫不仅包括上述的植食性和 捕食性慓虫的不同亚科, 还包括漂虫亚科。

\section{2 环境因子数据}

为了探究各省㼼虫体型特征的地理分异与环 境因子的关系, 本文选择了 6 个环境因子, 分别为 年均温(mean annual temperature, TEM)、最冷月均温 (mean temperature of the coldest month, TEMmin)、 年降雨量 (annual precipitation, PRE)、湿润指数 (moisture index, MI; 即AET/PET, 其中AET和PET 分别为实际蒸散量和潜在蒸散量)、年温度变化范围 (annual temperature range, TEMvar; 即最热月和最 冷月均温的差值)和年降水量变化范围(annual precipitation range, PREvar, 即降水量最多和最少月的 差值)。其中TEM和TEMmin代表温度, PRE和MI代 表水分, TEMvar和PREvar分别代表温度与降水的 季节性。由于初步分析显示瓢虫个体的平均大小未 如反贝格曼法则预测的那样随纬度升高而下降, 所 以我们没有考虑与生长季长度相关的因子。另外, 由于各省面积(AREA)差异较大, 因此将面积也纳 入到统计分析中, 以消除其可能的影响。

温度和降水等气候因子来自 New等(1999), AET和PET来自Ahn和Tateishi (1994)。数据提取是 利用ArcGIS 9.3将中国矢量地图切割成3,844个 0.5 。的像元, 提取每个像元中心点经纬度以及每个中 心点的环境数据。各省内各像元环境因子的均值代 表其平均环境条件(Qian, 2013)。

\section{3 统计分析}

采用Spearman相关性系数检验自变量之间的 相关性，采用基于普通最小二乘法(ordinary least squares, OLS)的一元线性回归模型检验各环境因子 对墂虫体型指标的解释能力。 
环境因子之间若具有强烈的共线性(附录1), 可 能导致回归模型的过度拟合(Dormann et al., 2013)。 因此, 基于各个环境因子对漂虫体型指标的解释能 力(附录2), 我们去除了Spearman相关系数大于 0.75 的1对环境因子中生态意义较弱(即解释力较低)的 1 个(Zhang et al., 2012; Dormann et al., 2013)。根据这 一标准, 对于体长和体宽, 全部墂虫选择的因子是 TEM和MI, 植食性漂虫选择的是 MI、TEMvar和 PREvar, 捕食性漂虫选择的是TEMmin和MI; 对于 长宽比, 所有类群选择的均为PRE。

然后, 我们根据Akaike信息量准则(Akaike Information Criterion, AIC), 为各个㼼虫体型指标篮 选出最优模型(AIC值最小)及其所包含的自变量 (Quinn \& Keough, 2002)。AIC指数在模型的精确性 和复杂性之间作出权衡, 可以通过其值大小从一系 列备选模型中篮选出最优模型(Quinn \& Keough, 2002)。除上述环境因子本身外, 我们在模型篮选过 程中还考虑了二次方、交互作用和面积因子 (AREA)。由于面积与一些因子具有显著的相关性, 可能影响体型与环境因子关系的分析, 为了去除其 影响, 我们将AREA作为所有模型篮选的必选自变 量。由于地理分布数据本身的空间自相关可能会增 加统计过程中犯I型错误的可能性(Diniz-Filho et al., 2003), 我们采用Dutilleul (1993)提出的方法, 重新 估计自由度和显著性。本文的主要统计分析在 Spatial Analysis in Macroecology (SAM, Rangel et al., 2010)中完成。

\section{2 结果}

\section{1 各省漂虫多样性分布}

不同省份漂虫属和种的丰富度差异较大。基于 我们所采用的数据, 无论全部漂虫, 还是植食性瓢 虫和捕食性漂虫, 属和种丰富度最低的省份均为青 海和宁夏, 而最高的则是台湾和云南。全部漂虫属 和种丰富度的各省平均值分别为 35.0 属和 100.8 种, 捕食性漂虫种类远多于植食性漂虫, 各省平均值分 别为13.1属和45.9种以及3.7属和13.7种(附录3)。

\section{2 瓢虫体型的空间格局}

在 28 个省份中, 全部漂虫平均体长为 $4.76 \pm$ $0.52 \mathrm{~mm}$, 小于植食性漂虫 $(7.2 \pm 0.70 \mathrm{~mm})$, 略大于 捕食性漂虫 $(4.48 \pm 0.56 \mathrm{~mm})$ 。平均体宽也呈现类似 的格局, 即植食性瓢虫体宽最大 $(5.62 \pm 0.55 \mathrm{~mm})$,
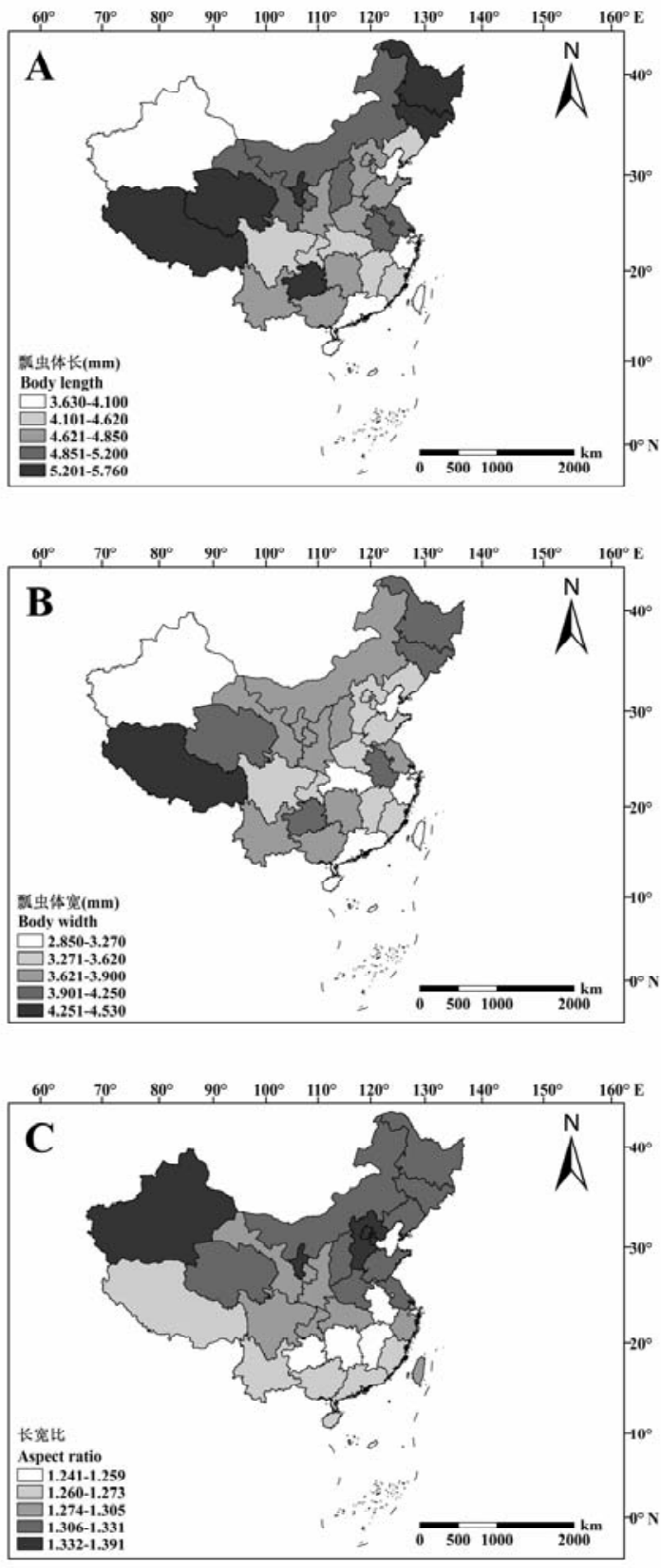

图1 中国全部瓢虫体长 $(\mathrm{A})$ 、体宽(B)和长宽比 $(\mathrm{C})$ 的省级分 布格局

Fig. 1 Spatial patterns of body length (A), body width (B) and aspect ratio (C) of all ladybirds at the provincial level

而全部瓢虫 $(3.66 \pm 0.41 \mathrm{~mm})$ 与捕食性慓虫 $(3.45 \pm$ $0.41 \mathrm{~mm})$ 间没有明显差异。各类慓虫长宽比没有显 著差异(全部慓虫 $1.30 \pm 0.04 \mathrm{~mm}$; 植食性慓虫 1.28 $\pm 0.06 \mathrm{~mm}$; 捕食性㼼虫 $1.30 \pm 0.04 \mathrm{~mm})$ 。

对全部㼼虫而言, 北方省份漂虫体长和长宽比 均较大, 而南方省份一般较小, 但体宽没有明显的 空间特征(图1), 即体长和长宽比与纬度呈显著正相 
关，而体宽与纬度的相关性不显著(图2A, B, C)。植 食性和捕食性漂虫的体长与体宽均与纬度呈显著 正相关(图2D，E)，而长宽比与纬度的关系明显具有
类群依赖性：即植食性漂虫长宽比与纬度没有显著 关系，而捕食性慓虫长宽比与纬度呈显著正相关 (图2F)。

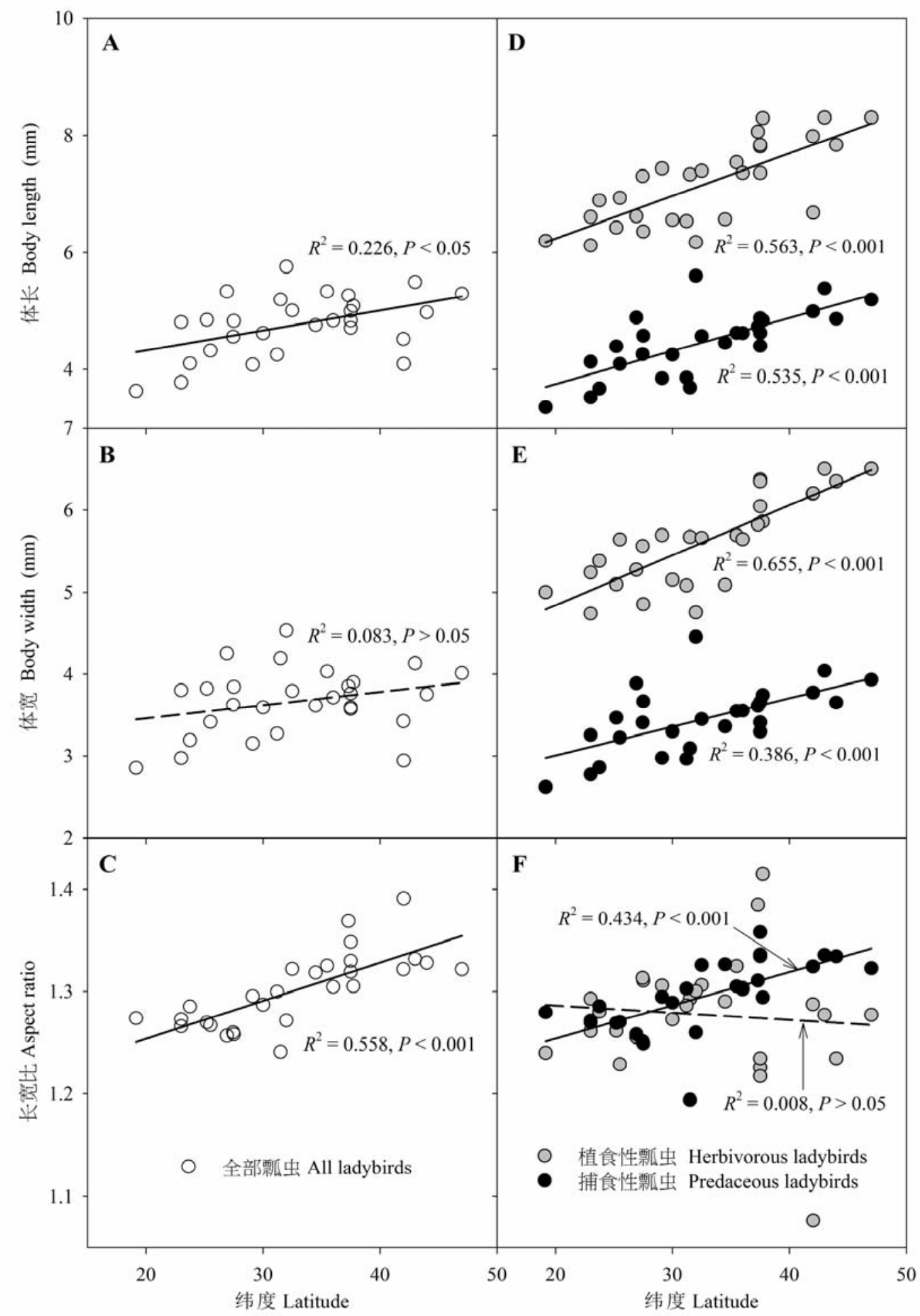

图2 不同类群瓢虫体长 $(\mathrm{A}, \mathrm{D})$ 、体宽 $(\mathrm{B}, \mathrm{E})$ 和长宽比 $(\mathrm{C}, \mathrm{F})$ 随纬度的变化趋势

Fig. 2 Latitudinal trends of body length (A, D), body width (B, E) and aspect ratio (C, F) of ladybirds 
表1 基于Akaike信息量准则(AIC)篮选出的瓢虫体型最优模型

Table 1 The best models selected for ladybird shape based on Akaike Information Criterion (AIC)

\begin{tabular}{|c|c|c|c|}
\hline $\begin{array}{c}\text { 因变量 } \\
\text { Response variables }\end{array}$ & $\begin{array}{l}\text { 预测因子 } \\
\text { Predictors }\end{array}$ & $\begin{array}{c}\text { 校正 } R^{2} \\
\text { Adjusted } R^{2}\end{array}$ & $P$ \\
\hline \multicolumn{4}{|l|}{ 全部慓虫 All ladybirds } \\
\hline 体长 Body length & TEM (-0.923), MI (+0.346), AREA (-0.049) & 0.462 & 0.011 \\
\hline 体宽 Body width & TEM (-0.795), MI (+0.488), AREA (+0.069) & 0.341 & 0.009 \\
\hline 长宽比 Aspect ratio & PRE (-0.972), AREA (-0.382) & 0.609 & 0.011 \\
\hline \multicolumn{4}{|l|}{ 植食性漂虫 Herbivorous ladybirds } \\
\hline 体长 Body length & TEMvar (+0.874), AREA (-0.324) & 0.645 & 0.005 \\
\hline 体宽 Body width & TEMvar (+0.847), MI (-0.237), AREA (-0.310) & 0.711 & 0.003 \\
\hline 长宽比 Aspect ratio & PRE $(-0.192)$, AREA $(+0.364)$ & 0.046 & 0.173 \\
\hline \multicolumn{4}{|l|}{ 捕食性慓虫 Predaceous ladybirds } \\
\hline 体长 Body length & TEMmin (-0.756), AREA (+0.119) & 0.682 & 0.005 \\
\hline 体宽 Body width & TEMmin (-0.623), AREA (+0.216) & 0.582 & 0.006 \\
\hline 长宽比 Aspect ratio & PRE $(-\mathbf{0 . 8 1 8})$, AREA $(+0.401)$ & 0.395 & 0.058 \\
\hline
\end{tabular}

括号内为标准回归系数, 其中统计上显著的以粗体表示。

Standardized regression coefficients were placed in the brackets, and the statistically significant ones are written in bold.

\section{3 体型指标与环境因子的关系}

对全部漂虫而言，由TEM、MI和AREA组成的 模型能够分别解释其体长和体宽空间分异的 $46.2 \%$ 和 $34.1 \%$ 。植食性慓虫体长和体宽的最优模型分别 包括TEMvar和AREA以及TEMvar、MI和AREA, 分 别能够解释其空间分异的 $64.5 \%$ 和 $71.1 \%$ 。捕食性漂 虫体长和体宽的最优模型均由 TEMmin和AREA组 成, 分别解释其空间分异的 $68.2 \%$ 和 $58.2 \%$ 。各类㼼 虫长宽比的最优模型只包括PRE和AREA, 对全部 漂虫长宽比的解释力最强(60.9\%), 其次是捕食性 㼼虫(39.5\%), 而对植食性瓢虫长宽比的解释力极 低(4.6\%) (表1)。

\section{3 讨论}

迄今为止, 脊椎动物体型大小的地理分异及其 机制研究较为充分(Chown \& Gaston, 2010)。虽然昆 虫的多样性远高于脊椎动物, 但很多类群体型特征 随纬度的变化符合贝格曼法则还是反贝格曼法则 仍然不明确, 更遑论其可能的生理、进化和环境制 约机制(Mousseau, 1997; Blanckenhorn \& Demont, 2004; Chown \& Gaston, 2010)。因此, 整合多源数据, 对不同的昆虫类群在各个尺度上检验其体型分异 格局及其与环境因子的关系, 是非常必要的(匡先 矩等, 2015)。

Shelomi (2012)总结了66例利用昆虫种间形态
数据检验贝格曼法则的结果, 发现 18 例支持贝格曼 法则, 12例支持反贝格曼法则, 多半(36例)缺乏体型 大小的纬度地带性, 且鞘翅目昆虫出现反贝格曼法 则的可能性更高。本文分析结果显示，虽然漂虫科 从属于鞘翅目，但除全部㼼虫的体宽外，植食性和 捕食性㼼虫的体长和体宽均存在显著的纬度地带 性, 即从低纬度到高纬度瓢虫的体长和体宽随之增 大，这一结果符合贝格曼法则。我们推测是因为我 国南方(低纬度)地区温度较高, 㼼虫的新陈代谢速 度较快, 生殖器官发育快, 成熟早, 生命周期短, 因而体型偏小。而北方(高纬度)地区气候寒冷, 影响 了体内酶的活性，新陈代谢慢，生命周期长，体内 营养物质积累多，因而体型偏大。

对各个类群而言, 影响其体长和体宽的最重要 环境因子均与温度相关, 支持基于温度的机理解 释，即高纬度地区漂虫个体增大是低温制约的结 果。首先, 年均温和最冷月均温都随着纬度的升高 而降低, 且均与温度季节性呈负相关, 更大的个体 对于寒冷地区的动物保持体温更有利。随着动物体 型的增大, 相对体表面积变小, 散失的热量变少, 有利于慓虫保持体温从而安全越冬。其次，随着纬 度的增加, 瓢虫要获得同样的食物, 需要付出更多 的能量, 如增加取食距离、延长取食时间, 其营养 生长的时间相应延长，导致个体较大(Yom-Tov \& Geffen, 2006)。更重要的是, 昆虫的脂肪含量与个体 
大小显著正相关, 更多的脂肪积累有利于昆虫越 冬。昆虫在低温暴露期间, 基本依靠脂肪产生维持 新陈代谢的能量(Renault et al., 2003)。而饥力假说 认为, 体型越大, 则能量储存越多, 而饥力随之增 强(Arnett \& Gotelli, 2003)。而慓虫以成虫越冬, 增 加能量储存以提高耐饥力这一机制尤为重要(赵静 等, 2010)。

在各个纬度上, 植食性慓虫均比捕食性慓虫更 大, 表现在体长和体宽均大; 虽然在各个纬度上长 宽比没有显著差异, 但捕食性慓虫的长宽比随纬度 升高显著增大, 而植食性漂虫不然。我们推测其原 因可能在于其营养级、取食方式和环境因子的制 约。由于食性不同, 捕食性昆虫体内的氮含量高于 植食性昆虫(Fagan et al., 2002; Martinson et al., 2008), 植食性昆虫需要通过增加取食或减缓生长 发育以获取足够的营养元素, 从而导致体型增大。

捕食性漂虫的长宽比具有显著的空间分异, 即 随纬度升高而增加, 但植食性漂虫则不然。这表明 全部㼼虫长宽比的纬度地带性主要是由物种比例 较高的捕食性瓢虫导致的; 同时也说明, 飞行能力 对于捕食性漂虫而言更是一个适应性状, 因为其食 物的空间分布可能更为分散。植食性漂虫(食植漂虫 亚科)主要取食茄科、葫芦科、菊科、豆科、禾本科 等广泛分布的植物(庞虹, 2001); 而捕食性慓虫的 取食对象包括蚜虫、介壳虫、粉䖝和叶螨等, 且不 同亚科漂虫具有一定的专食对象(王兴民等, 2014)。 捕食对象, 如蚜虫类也有很高的寄主植物特异性, 很多种类只寄生在一种或几种寄主植物上(黄晓磊 和乔格侠, 2006)。捕食性㼼虫食物的分布不仅受制 于捕食对象本身的分布, 而且还受捕食对象寄主植 物分布的影响，因此其食物的分布更为分散。

以往研究认为, 展弦比随纬度增加主要是弥补 低温对昆虫翅膀振动频率降低的影响(Azevedo et al., 1998; Blanckenhorn \& Demont, 2004; Vandewoestijne \& Van Dyck, 2011)。但我们的研究结果表 明, 作为展弦比的粗略度量, 长宽比主要受年降水 量的影响。年降水量降低导致植被斑块化程度增加, 使得捕食性漂虫的食物分布更为分散。因此长宽比 随年降水量降低而增加是一种适应性变化, 可以提 高其飞行能力和捕食能力。捕食性漂虫的活动和取 食行为与植食性瓢虫差异较大, 可能需要更强的飞 行能力, 因此, 捕食性瓢虫的长宽比具有纬度地带
性, 且与年降水量显著相关。另外, 我们的结果表 明, 气候变化(特别是温度升高)不仅对动物体型大 小有影响(Sheridan \& Bickford, 2011), 还可能影响 其形状, 而且这些影响具有一定的类群依赖性。

由于数据的缺乏, 我们只能依靠有限的体型和 分布数据, 在较粗的空间尺度上(省级行政区)分析 慓虫体型的地理分异及其与环境因子的关系。因此, 本文结果的可靠性仍然需要在不同尺度(如群落)、 生境以及种内进行检验。近年发展迅速的公众科学 项目有可能为慓虫及其他昆虫类群的生物地理学 研究提供诸如数据收集与分析方面的支持(张健等, 2013)。

\section{参考文献}

Ahn CH, Tateishi R (1994) Development of a global 30-minute grid potential evapotranspiration data set. Photogrammetry and Remote Sensing, 33, 12-21.

Allen JA (1877) The influence of physical conditions in the genesis of species. Radical Review, 1, 108-140.

Arnett AE, Gotelli NJ (2003) Bergmann's rule in larval ant lions: testing the starvation resistance hypothesis. Ecological Entomology, 28, 645-650.

Azevedo RBR, James AC, McCabe J, Partridge L (1998) Latitudinal variation of wing: thorax size ratio and wing-aspect ratio in Drosophila melanogaster. Evolution, 52, 1353-1362.

Barbault R (1988) Body size, ecological constraints, and the evolution of life-history strategies. Evolutionary Biology, 22, 261-286.

Bergmann C (1847) Ueber die Verhältnisse der Wärmeökonomie der Thiere zu ihrer Grösse. Gottinger Studien, 3, 595708.

Blackburn TM, Gaston KJ, Loder N (1999) Geographic gradients in body size: a clarification of Bergmann's rule. Diversity and Distributions, 5, 165-174.

Blanckenhorn WU, Demont M (2004) Bergmann and converse Bergmann latitudinal clines in arthropods: two ends of a continuum? Integrative and Comparative Biology, 44, 413424.

Brown JH, Gillooly JF, Allen AP, Savage VM, West GB (2004) Toward a metabolic theory of ecology. Ecology, 85, 1771-1789.

Chown SL, Gaston KJ (2010) Body size variation in insects: a macroecological perspective. Biological Reviews, 85, 139-169.

Comont RF, Roy HE, Lewis OT, Harrington R, Shortall CR, Purse BV (2012) Using biological traits to explain ladybird distribution patterns. Journal of Biogeography, 39, 17721781.

Diniz-Filho JAF, Bini LM, Hawkins BA (2003) Spatial autocorrelation and red herrings in geographical ecology. Global 
Ecology and Biogeography, 12, 53-64.

Dormann CF, Elith J, Bacher S, Buchmann C, Carl G, Carré G, Marquéz JRG, Gruber B, Lafourcade B, Leitão PJ, Münkemüller T, McClean C, Osborne PE, Reineking B, Schröder B, Skidmore AK, Zurell D, Lautenbach S (2013) Collinearity: a review of methods to deal with it and a simulation study evaluating their performance. Ecography, 36, 27-46.

Dutilleul P (1993) Modifying the $t$ test for assessing the correlation between two spatial processes. Biometrics, 49, 305-314.

Fagan WF, Siemann E, Mitter C, Denno RF, Huberty AF, Woods HA, Elser JJ (2002) Nitrogen in insects: implications for trophic complexity and species diversification. The American Naturalist, 160, 784-802.

Gardner JL, Peters A, Kearney MR, Joseph L, Heinsohn R (2011) Declining body size: a third universal response to warming. Trends in Ecology and Evolution, 52, 285-291.

Gruner DS (2003) Regressions of length and width to predict arthropod biomass in the Hawaiian Islands. Pacific Science, 57, 325-336.

Hoffmann AA, Shirriffs J (2002) Geographic variation for wing shape in Drosophila serrata. Evolution, 56, 1068-1073.

Huang XL (黄晓碟), Qiao GX (乔格侠) (2006) Research status and trends in aphidology. Acta Entomologica Sinica (昆虫 学报), 49, 1017-1026. (in Chinese with English abstract)

Kuang XJ (匡先矩), Ge F (戈峰), Xue FS (薛芳森) (2015) Geographic variation in body size and sexual dimorphism in insects. Acta Entomologica Sinica (昆虫学报), 58, 351-360. (in Chinese with English abstract)

Martinson HM, Schneider K, Gilbert J, Hines JE, Hambäck PA, Fagan WF (2008) Detritivory: stoichiometry of a neglected trophic level. Ecological Research, 23, 487-491.

Masakis S (1967) Geographic variation and climatic adaptation in a field cricket (Orthoptera: Gryllidae). Evolution, 21, 725-741.

Mousseau TA (1997) Ectotherms follow the converse to Bergmann's rule. Evolution, 51, 630-632.

New M, Hulme M, Jones P (1999) Representing twentieth-century space-time climate variability. Part I. Development of a 1961-90 mean monthly terrestrial climatology. Journal of Climate, 12, 829-856.

Nylin S, Svärd L (1991) Latitudinal patterns in the size of European butterflies. Ecography, 14, 192-202.

Pang H (庞虹) (2001) Current situation of the systematics of Coccinellidae. Entomological Knowledge (昆虫知识), 39, 17-22. (in Chinese with English abstract)

Pang H (庞虹), Ren SX (任顺祥), Zeng T (曾涛), Pang XF (庞 雄飞) (2004) Biodiversity and Their Utilization of Coccinellidae in China (中国㼼虫物种多样性及其利用). Guangdong Science and Technology Press, Guangzhou. (in Chinese)

Park O (1949) Application of the converse Bergmann principle to the carabid beetle, Dicaelus purpuratus. Physiological
Zoology, 22, 359-372.

Peters R (1983) Nuclear envelope permeability measured by fluorescence microphotolysis of single liver cell nuclei. Journal of Biological Chemistry, 258, 11427-11429.

Qian H (2013) Environmental determinants of woody plant diversity at a regional scale in China. PLoS ONE, 8, e75832.

Quinn GP, Keough MJ (2002) Experimental Design and Data Analysis for Biologists. Cambridge University Press, Cambridge.

Rangel TF, Diniz-Filho JAF, Bini LM (2010) SAM: a comprehensive application for spatial analysis in macroecology. Ecography, 33, 46-50.

Ren SX (任顺祥), Wang XM (王兴民), Pang H (庞虹), Peng ZQ (彭正强), Zeng T (曾涛) (2009) Colored Pictorial Handbook of Ladybird Beetles (中国㼼虫原色图鉴). Science Press, Beijing. (in Chinese)

Renault D, Hance T, Vannier G, Vernon P (2003) Is body size an influential parameter in determining the duration of survival at low temperatures in Alphitobius diaperinus Panzer (Coleoptera: Tenebrionidae)? Journal of Zoology, 259, 381-388.

Rogers LE, Hinds WT, Buschbom RL (1976) A general weight vs. length relationship for insects. Annals of the Entomological Society of America, 69, 387-389.

Shelomi M (2012) Where are we now? Bergmann's rule sensu lato in insects. The American Naturalist, 180, 511-519.

Sheridan JA, Bickford D (2011) Shrinking body size as an ecological response to climate change. Nature Climate Change, 1, 401-406.

Vandewoestijne S, Van Dyck H (2011) Flight morphology along a latitudinal gradient in a butterfly: Do geographic clines differ between agricultural and woodland landscapes? Ecography, 34, 876-886.

Wang XM (王兴民), Chen XS (陈晓胜), Qiu BL (邱宝利), Ren SX (任顺祥) (2014) Methods and technologies for collecting, surveying and sampling predatory ladybirds (Coleoptera: Coccinellidae). Chinese Journal of Applied Entomology (应用昆虫学报), 51, 1362-1366. (in Chinese with English abstract)

Yom-Tov Y, Geffen E (2006) Geographic variation in body size: the effects of ambient temperature and precipitation. Oecologia, 148, 213-218.

Zhang J (张健), Chen SB (陈圣宾), Chen B (陈涁), Du YJ (杜 彦君), Huang XL (黄晓否), Pan XB (潘旭斌), Zhang Q (张 强) (2013) Citizen science: integrating scientific research, ecological conservation and public participation. Biodiversity Science (生物多样性), 21, 738-749. (in Chinese with English abstract)

Zhang MG, Zhou ZK, Chen WY, Slik JWF, Cannon CH, Raes N (2012) Using species distribution modeling to improve conservation and land use planning of Yunnan, China. Biological Conservation, 153, 257-264.

Zhao J (赵静), Cui NN (崔宁宁), Zhang F (张帆), Yin XC (印 
象初), Xu YY (许永玉) (2010) Effects of body size and fat content on cold tolerance in adults of Harmonia axyridis (Pallas) (Coleoptera: Coccinellidae). Acta Entomologica
Sinica (昆虫学报), 52, 1213-1219. (in Chinese with English abstract)

(责任编委: 黄晓䂞 责任编辑: 闵文杰)

\section{附录 Supplementary Material}

附录1 环境因子之间的Spearman相关系数

Appendix 1 Spearman correlation coefficients between environmental variables http://www.biodiversity-science.net/fileup/PDF/w2015-076-1.pdf

附录2 各个环境因子对漂虫体长、体宽和长宽比的解释力

Appendix 2 The explanatory power of each environmental variable for body length, body width and aspect ratio http://www.biodiversity-science.net/fileup/PDF/w2015-076-2.pdf

附录3 具有体型数据的漂虫属、种丰富度在各省的分布概况

Appendix 3 The numbers of ladybird genera and species in each China's province http://www.biodiversity-science.net/fileup/PDF/w2015-076-3.pdf 
毕孟杰, 沈梦伟, 周可新, 毛岭峰, 陈圣宾, 彭培好. 中国漂虫体型的地理分异及其与环境因子的关系. 生物多样性, 2015, 23(6)：775-783.

http://www. biodiversity-science. net/CN/10. 17520/biods. 2015076

附录1 7 个环境因子之间的Spearman相关系数

Appendix 1 Spearman correlation coefficients between environmental variables

\begin{tabular}{|c|c|c|c|c|c|c|}
\hline 变量 Variables & 面积 AREA & 年均温 TEM & 最冷月均温 TEMmin & 年降雨量 PRE & 湿润指数 MI & 年温度变化范围 TEMvar \\
\hline 年均温 TEM & $-0.533^{* *}$ & & & & & \\
\hline 最冷月均温 TEMmin & $-0.488^{*}$ & $0.977^{* * *}$ & & & & \\
\hline 年降雨量 PRE & $-0.536^{* *}$ & $0.928^{* * *}$ & $0.919^{* * *}$ & & & \\
\hline 湿润指数 MI & $-0.414^{*}$ & $0.706^{*}$ & 0.651 & $0.825^{* *}$ & & \\
\hline 年温度变化范围 TEMvar & 0.209 & $-0.758^{*}$ & $-0.857^{* *}$ & $-0.786^{*}$ & -0.475 & \\
\hline 年降水量变化范围 PREvar & $-0.532 * *$ & $0.835^{* *}$ & $0.822^{* *}$ & $0.907^{* * * *}$ & $0.667^{* *}$ & $-0.667^{* *}$ \\
\hline
\end{tabular}

采用Dutilleul (1993)的方法去除空间自相关后检验相关性的显著性。The $P$ values were calculated after accounting for spatial autocorrelation using Dutilleul’s (1993) method.

* $P<0.05 ; * * P<0.01 ; * * * P<0.001$ 。

年均温(mean annual temperature, TEM)、最冷月均温(mean temperature of the coldest month, TEMmin)、年降雨量(annual precipitation, PRE)、湿润指数(moisture index, MI; 即年实际蒸发量AET/年 潜在蒸发量PET)、年温度变化范围(annual temperature range, TEMvar; 即最热月和最冷月均温的差值)和年降水量变化范围(annual precipitation range, PREvar, 即降水量最多和最少月的差值)。其 中TEM和TEMmin代表温度, PRE和MI代表水分, TEMvar和PREvar分别代表温度与降水的季节性。 
毕孟杰, 沈梦伟, 周可新, 毛岭峰, 陈圣宾, 彭培好. 中国慓虫体型的地理分异及其与环境因子的关系. 生物多样性, 2015, 23(6)：775-783.

http://www. biodiversity-science. net/CN/10. 17520/biods. 2015076

附录2 各个环境因子对㼼虫体长、体宽和长宽比的解释能力

Appendix 2 The explanatory power of each environmental variable for body length, body width and aspect ratio

\begin{tabular}{|c|c|c|c|c|c|c|c|}
\hline & 面积 AREA & 年均温 TEM & 最冷月均温 TEMmin & 年降雨量 PRE & 湿润指数 MI & 年温度变化范围 TEMvar & 年降水量变化范围 PREvar \\
\hline \multicolumn{8}{|l|}{ 体长 Body length } \\
\hline 全部慓虫 All ladybirds & 0.144 & $0.432^{*}$ & $0.382^{*}$ & 0.354 & 0.062 & 0.198 & 0.251 \\
\hline 植食性慓虫 Herbivorous ladybird & 0.000 & 0.300 & $0.462^{*}$ & 0.321 & 0.077 & $0.567^{*}$ & 0.214 \\
\hline 捕食性漂虫 Predaceous ladybird & $0.331 *$ & $0.666^{* * * *}$ & $0.685^{* *}$ & $0.586^{*}$ & 0.238 & $0.453^{*}$ & $0.404 *$ \\
\hline \multicolumn{8}{|l|}{ 体宽 Body width } \\
\hline 全部慓虫 All ladybirds & 0.112 & 0.263 & 0.204 & 0.172 & 0.007 & 0.079 & 0.118 \\
\hline 植食性漂虫 Herbivorous ladybird & 0.018 & 0.277 & $0.486^{* *}$ & 0.361 & 0.130 & $0.662^{*}$ & 0.211 \\
\hline 捕食性慓虫 Predaceous ladybird & $0.351 *$ & $0.594^{* *}$ & $0.568^{* *}$ & $0.470^{*}$ & 0.185 & 0.328 & 0.351 \\
\hline \multicolumn{8}{|l|}{ 长宽比 Aspect ratio } \\
\hline 全部慓虫 All ladybirds & 0.053 & 0.326 & $0.435^{*}$ & $0.535^{*}$ & $0.472 *$ & $0.425^{*}$ & $0.427 *$ \\
\hline 植食性慓虫 Herbivorous ladybird & 0.059 & 0.005 & 0.000 & 0.019 & 0.001 & 0.008 & 0.002 \\
\hline 捕食性慓虫 Predaceous ladybird & 0.013 & 0.190 & 0.298 & 0.320 & 0.154 & 0.352 & 0.139 \\
\hline
\end{tabular}

采用Dutilleul (1993)的方法去除空间自相关后检验显著性。The $P$ values were calculated after accounting for spatial autocorrelation using Dutilleul (1993)'s method. ${ }^{*} P<0.05$; $* * P<0.01$ 。 
毕孟杰, 沈梦伟, 周可新, 毛岭峰, 陈圣宾, 彭培好. 中国慓虫体型的地理分异及其与环境因子的关系. 生物多样性, 2015, 23 (6)：775-783. http://www. biodiversity-science. net/CN/10. 17520/biods. 2015076

附录3 具有体型数据的慓虫属、种丰富度在各省的分布概况

Appendix 3 The numbers of ladybird genera and species in each of China's province

\begin{tabular}{|c|c|c|c|c|c|c|c|c|c|c|c|c|c|c|c|}
\hline \multirow[t]{2}{*}{ 省份 Province } & \multicolumn{5}{|c|}{ 植食性慓虫 Herbivorous ladybirds } & \multicolumn{5}{|c|}{ 捕食性慓虫 Predaceous ladybirds } & \multicolumn{5}{|c|}{ 全部漂虫 All ladybirds } \\
\hline & 属数(A) & 种数(B) & 体长(C) & 体宽(D) & 长宽比(E) & 属数(A) & 种数(B) & 体长(C) & 体宽(D) & 长宽比(E) & 属数(A) & 种数(B) & 体长(C) & 体宽(D) & 长宽比(E) \\
\hline 安徽 Anhui & 4 & 8 & 7.33 & 5.67 & 1.29 & 7 & 12 & 3.69 & 3.09 & 1.19 & 21 & 32 & 5.20 & 4.19 & 1.24 \\
\hline 福建 Fujian & 4 & 13 & 6.93 & 5.64 & 1.23 & 22 & 116 & 4.09 & 3.22 & 1.27 & 49 & 184 & 4.32 & 3.41 & 1.27 \\
\hline 甘肃 Gansu & 3 & 8 & 8.29 & 5.86 & 1.41 & 12 & 32 & 4.84 & 3.74 & 1.29 & 36 & 88 & 5.09 & 3.90 & 1.31 \\
\hline 广东 Guangdong & 5 & 20 & 6.12 & 4.74 & 1.29 & 25 & 131 & 3.52 & 2.77 & 1.27 & 53 & 202 & 3.78 & 2.97 & 1.27 \\
\hline 广西 Guangxi & 6 & 39 & 6.61 & 5.24 & 1.26 & 18 & 65 & 4.13 & 3.25 & 1.27 & 45 & 153 & 4.81 & 3.80 & 1.27 \\
\hline 贵州 Guizhou & 5 & 28 & 6.62 & 5.27 & 1.26 & 14 & 49 & 4.89 & 3.88 & 1.26 & 40 & 126 & 5.34 & 4.25 & 1.26 \\
\hline 海南 Hainan & 4 & 14 & 6.20 & 5.00 & 1.24 & 26 & 102 & 3.35 & 2.62 & 1.28 & 48 & 154 & 3.63 & 2.85 & 1.27 \\
\hline 河北 Hebei & 3 & 6 & 7.81 & 6.37 & 1.23 & 10 & 32 & 4.62 & 3.41 & 1.36 & 30 & 79 & 4.84 & 3.59 & 1.35 \\
\hline 黑龙江 Heilongjiang & 2 & 2 & 8.30 & 6.50 & 1.28 & 7 & 12 & 5.20 & 3.93 & 1.32 & 25 & 42 & 5.30 & 4.01 & 1.32 \\
\hline 河南 Henan & 4 & 9 & 6.56 & 5.09 & 1.29 & 13 & 38 & 4.45 & 3.36 & 1.33 & 34 & 82 & 4.76 & 3.61 & 1.32 \\
\hline 湖北 Hubei & 4 & 12 & 6.53 & 5.08 & 1.29 & 15 & 54 & 3.86 & 2.96 & 1.30 & 35 & 93 & 4.25 & 3.27 & 1.30 \\
\hline 湖南 Hunan & 4 & 9 & 6.36 & 4.85 & 1.31 & 11 & 29 & 4.58 & 3.66 & 1.25 & 32 & 72 & 4.83 & 3.84 & 1.26 \\
\hline 江苏 Jiangsu & 3 & 8 & 7.39 & 5.66 & 1.31 & 7 & 21 & 4.57 & 3.45 & 1.33 & 24 & 52 & 5.01 & 3.79 & 1.32 \\
\hline 江西 Jiangxi & 3 & 6 & 7.30 & 5.56 & 1.31 & 10 & 24 & 4.26 & 3.40 & 1.25 & 28 & 57 & 4.56 & 3.62 & 1.26 \\
\hline 吉林 Jilin & 2 & 2 & 8.30 & 6.50 & 1.28 & 5 & 11 & 5.39 & 4.04 & 1.34 & 24 & 40 & 5.50 & 4.13 & 1.33 \\
\hline 辽宁 Liaoning & 3 & 3 & 7.98 & 6.20 & 1.29 & 8 & 15 & 4.99 & 3.77 & 1.32 & 25 & 38 & 4.52 & 3.42 & 1.32 \\
\hline 内蒙古 Neimonggu & 2 & 3 & 7.84 & 6.35 & 1.23 & 8 & 22 & 4.87 & 3.65 & 1.33 & 28 & 68 & 4.98 & 3.75 & 1.33 \\
\hline 宁夏 Ningxia & 1 & 1 & 8.06 & 5.82 & 1.39 & 4 & 7 & 4.73 & 3.61 & 1.31 & 17 & 28 & 5.27 & 3.85 & 1.37 \\
\hline 青海 Qinghai & 1 & 1 & 7.54 & 5.69 & 1.32 & 3 & 5 & 4.62 & 3.54 & 1.31 & 15 & 24 & 5.34 & 4.03 & 1.33 \\
\hline 陕西 Shaanxi & 4 & 9 & 7.36 & 5.64 & 1.30 & 17 & 45 & 4.62 & 3.55 & 1.30 & 42 & 118 & 4.84 & 3.71 & 1.30 \\
\hline 山东 Shandong & 4 & 6 & 7.36 & 6.04 & 1.22 & 10 & 25 & 4.40 & 3.29 & 1.34 & 29 & 61 & 4.71 & 3.57 & 1.32 \\
\hline 山西 Shanxi & 2 & 3 & 7.84 & 6.35 & 1.23 & 10 & 22 & 4.88 & 3.65 & 1.33 & 30 & 58 & 5.00 & 3.76 & 1.33 \\
\hline 四川 Sichuan & 7 & 31 & 6.56 & 5.15 & 1.27 & 20 & 82 & 4.25 & 3.30 & 1.29 & 55 & 195 & 4.62 & 3.59 & 1.29 \\
\hline 台湾 Taiwan & 5 & 50 & 6.89 & 5.38 & 1.28 & 32 & 125 & 3.67 & 2.86 & 1.29 & 61 & 246 & 4.10 & 3.19 & 1.29 \\
\hline 新疆 Xinjiang & 2 & 2 & 6.68 & 6.20 & 1.08 & 8 & 17 & 5.00 & 3.77 & 1.32 & 24 & 56 & 4.09 & 2.94 & 1.39 \\
\hline 西藏 Xizang & 7 & 25 & 6.18 & 4.75 & 1.30 & 8 & 15 & 5.61 & 4.45 & 1.26 & 39 & 103 & 5.76 & 4.53 & 1.27 \\
\hline 云南 Yunnan & 7 & 59 & 6.43 & 5.09 & 1.26 & 24 & 127 & 4.39 & 3.46 & 1.27 & 60 & 286 & 4.85 & 3.82 & 1.27 \\
\hline 浙江 Zhejiang & 3 & 6 & 7.43 & 5.69 & 1.31 & 13 & 50 & 3.85 & 2.97 & 1.29 & 31 & 85 & 4.08 & 3.15 & 1.30 \\
\hline 平均值 Mean value & 3.7 & 13.7 & 7.2 & 5.62 & 1.28 & 13.1 & 45.9 & 4.48 & 3.45 & 1.30 & 35.0 & 100.8 & 4.76 & 3.66 & 1.30 \\
\hline
\end{tabular}

A: 属数; B: 种数; C: 体长; D: 体宽; E: 长宽比

A, No. of genus; B, No. of species; C, Body length (mm); D, Body width (mm); E, Aspect ratio 